\title{
Discussion on the Existing Problems and Design Methods of Middle and Small Houses Architectural Design
}

\author{
Chunfang Xiang \\ Wuchang Institute of Technology, Wuhan Hubei, 430065, China
}

Keyword: Middle and small housing, Architectural design, Problems, Design method.

\begin{abstract}
With the continuous development of China's social economy and the continuous reform and innovation of housing system in recent years, China's realty business got an unprecedented development, the masses' living standard and housing conditions also got a quality improvement. Though housing has obtained many achievements, because of the aggravated population problem, China's realty business faced problems such as low greening rate, high building density and son so, which cause many difficulties for housing design and architecture. Middle and small housing is Chinese' main choice, it is also the benefit direction for reality business, therefore, architectural designer should try to make whole housing design be scientific and rational, and maximize the use of limited area and space, try to create multifunctional good quality housing, help to improve people's living quality and promote the good development of realty business.
\end{abstract}

\section{Introduction}

In recent years, real estate developers broaden residential given areas through the way of "touch ball" ${ }^{[1]}$, while government relevant departments have already controlled comprehensively. China has the biggest population in the world, and so, housing problems emerge in endlessly, even become even more violent. For the sound development of socialist social construction and people's happy life, this paper makes a deep analysis on the existing problems and design methods of middle and small houses architectural design to provide reference for the optimization of residential construction.

\section{Current situation of China's middle and small housing architectural design}

From the comprehensive perspective, many factors have different influence on housing quality, of which, residential environment, flat planning design and housing area are most important factors.

Generally speaking, residential environment is the key consideration for people to choose their residence, while the green rate, plot ratio and housing density of the residential area are the most important factors affecting the residential environment. On the premise of residential building height limit, residential plot ratio and residential density are in direct proportion, meanwhile, residential plot ratio and green rate are in inverse ratio, accordingly cause a certain adverse effect for the residential environment. As the earth's population continues to grow, human being's demand for housing is also increasingly expanded, for benefit, many real estate developers try their best to maximize the plot ratio, which caused high building density, crowd and depression gradually reduced the living and residential quality. Of which, residential areas with middle and small housing have bigger negative effects on their residential environment for their high plot ratio and building density. Therefore, under this situation, architectural designers should continue to optimize overall planning, on the basis of ensuring building land exploitation level to maximize green rate. Making spatial relationship of residential area be more humanized and making it closer to natural environment is the basic premise to build a high quality house. 
Model and area of the residence is the second biggest consideration for people buying a house. In the whole marketing process of real estate development projects, sales price for a single house will be correspondingly controlled, and because real estate sales price in recent years has always been in a steady upward trend, therefore, it is necessary to strictly control the dwelling size area. In order to further shrink dwelling size area on the basis of ensuring residence quality, the following principles must be abided by: (1) try multiple stratification reduce high-rise, meanwhile, try to minimize shared area; (2) try to retionalise residential ratio, especially for middle and small housing, try to develop to several flats with one staircase, gradually decrease housing type that two families share a ladder, if necessary (In some special cases), can consider five families share a ladder. In general, try to apply limited land buildings resource into every house, to broaden housing space at the same time with design high-end residence.

On the premise that residential environment and dwelling size area have been certain, good residential type design is the third consideration for people buying a house, while good residential type design general can attract customer to a greatly extent. Effective space is too narrow (or incomplete) or ineffective space is too huge are defects of many current commercial residential buildings. Relevant research found that dwelling type designers did not realize the realization and adaptation is a main reason for this problem. Housing type design must be based on human actual residential situation (law of human life and son on), and strictly control the space, meanwhile, must reasonably layout to ensure the completeness of the residential space. And because the landscape view and lighting area of every house is limited, therefore when design the dwelling type, designer must abide by overall planning, comprehensively consider view, ventilation, lighting and other factors and conditions. Designers with strong profession generally can maximize space of every house, extend under the limited condition to design comprehensive high quality house.

\section{Current existing problems of middle and small housing architectural design}

The people's living standards rises along with the economic development, besides food and clothing this basic need, people's requirements on residential environment and quality also rises. Real estate developers want to stand firm and obtain more economic benefit under current market economy condition, they first need to take "low residential plot ratio \& building density and high green rate ${ }^{[2] "}$ "as new building orientation. But for the moment, if the single building area is two small, then on the premise that plot ratio do not change, the building floors and number of buildings will increase, which means the distance among buildings will become smaller and smaller. Meanwhile, designers also should consider daylighting standard of different areas to ensure building can reach the national minimum sunshine requirement, therefore, they must strictly control the building numbers and floors further to guarantee the balanced development.

From the most simple residence, if according to middle and high floors to design can effectively reduce building numbers and floor density to a certain extent, but if the project is mainly high-rise housing, plot ratio becomes the primary consideration factor. From the relative point of view, fire protection criteria of medium and high-rise housing is low, but the traffic area in internal public area is normally small. Two families share a ladder is main type of big housing, while the floor discretion is the key point of building distance in this kind of project (the two is in direct ratio). When develop the project with low plot ratio, designers should continue to reach the construction requirement of low plot ratio and high green rate. Meanwhile, real estate project with low plot ratio generally has low technical requirement, therefore almost did not exist technical matters.

Population increases sharply but with deficient land source, this is a fundamental reality of the country, on the way of socialist construction, saving land resource is a fundamental policy for all regional government. In the urban construction, in order to further meet needs of vulnerable groups with low economic strength, small and medium dwelling unit type occupies a certain proportion in the government tender documents. Therefore, how to ensure the comprehensive quality of residence 
and effectively solve plot ratio and green rate problems becomes the headache for real estate developers.

\section{Design method for middle and small housing}

\section{The total plane distribution}

Build better quality house for the mass under the architectural concept of low plot ratio and high green rate is the basic target for construction project. In order to further implement the construction goal, real estate developers should start from following points:

Firstly, try to reduce building numbers. Designers should scientifically and rationally broaden single floor area, try to design 6 families in each building floor, further to improve building design and layout flexibility. Therefore, they can not only broaden single floor area, but also can greatly reduce shared area ratio.

Secondly, increase building floors within the reasonable building height scope and rationalize building-higher. If can continue to reduce about $2 \mathrm{~cm}$ on the original height of each floor, thus this building can get 1-2 floors building area. Compared with broaden single floor building area, increasing floors is more advantage, because sacrifice residential comprehensive quality is the cost for broaden single floor building area and reduce floor height. In some ways, per capita building area is in inverse ration with total family number, increase of planning index is the necessary trend for increasing total residential number. Parking space increasing is one of the most important problems faced by real estate developers. Continuously expand of parking space will bring adverse effect to residential environment, continues to reduce living quality. Assisted by ground parking, designers try to minimize adverse effect of vehicles on people's living.

\section{A comprehensive design analysis on building unit}

Discussion on shared proportion of building area

Compared with sensitive home buyers, rational buyers pay more attention to shared proportion. From the practical point of view, shared proportion below $15.00 \%$ can be accepted, if it is over $20.00 \%$, it is hard to be accepted. High-rise building over 15 floors, housing type that four families share a ladder, the public area is around $42 \sim 71 \mathrm{~m}^{2[3]}$. Relevant researches in our country found that six families share a ladder is the best choice for control shared areas for housing type below $90 \mathrm{~m}^{2}$.

Discussion on refine housing type design

Bedroom, kitchen, washroom, living room and balcony are main components of modern house, scientifically and reasonably combine these parts can ensure the rationality of segregation of living area from common activity area. From the broad sense and practical perspective, based on different product positioning and customers' specific requirement to do targeted design is necessary. Under the requirement of relevant national policies, if all kinds of housing design can just meet this requirement $=$, which is not enough, but require designers to design good living space in the limited area, which is a challenge and good opportunity for designers. In this process, designers should combine ergonomics to make a comprehensive thinking on the whole design, and consider different factors to make every space get a fine design to ensure flexibility and high quality.

Avoid ineffective space

With our country successively bring out all kinds of rules and regulations, the high-profile design concept "mansion ${ }^{[4]}$ "has become into a new concept of comfortable and freedom. In order to avoid defects of "mansion"in the design, designers should remove ineffective space (residential space be shrink but former functions will not remove) first.

Bedroom: in housing design, for bedroom, besides considering apace for bed, night table, designers also should consider bureau, dresser and other furniture. If the bedroom space is narrow, designers should try to design by-window to broaden space and open view. 
Kitchen: combination of kitchen and dining hall not only can shorten their distance, it is also convenient for residents' daily life, and upright and foursquare kitchen space is more suitable for placing tableware, and can further shrink ineffective space.

Washroom: in the design process, washroom function must be completed. In real life, many residents cannot accept toilet in front of door, but if do not take this design, the ineffective design will increase. Therefore, in actual design, insist to scientific design or according to customers' favor is a problem for designers.

Living room: relatively speaking, living room is the best choice for shrink ineffective space, and wall hung electric appliance has become a trend. Scientific research showed that $3.55 \mathrm{~m}$ is the best TV distance, while the seeing and hearing space in the living room should be between 3 and 4 meters. Therefore, reasonably shrink living room area not only can keep seeing and hearing function but also can greatly reduce ineffective space.

Balcony: for balcony design, besides considering basic sun-curing and pot culture function, also should consider its own space design to improve living quality to meet residents' requirement.

\section{Conclusion}

For real estate industry in the quality era, continue to increase selling points of commercial housing can further to keep its stable development. Therefore, under the influence of many factors especially relevant national policies, residential design aspiration and innovation spirit should be improved continue, which requires designers continue to improve their own comprehensive quality to create better environment for people.

\section{References}

[1] Lin Yahong. Discussion on the Existing Problems and Design Methods of Middle and Small Houses Architectural Design, Henan Building Materials, 2016,(05) : 141-142.

[2] Yang Jing. Discussion on the Existing Problems and Design Methods of Middle and Small Houses Architectural Design, Fujian Building Materials, 2015,(03) : 27-28.

[3] Liu Huolian. Brief Analysis on the Architectural Design for Middle and Small Housing, Fujian Building Materials, 2016, (10) : 38-39.

[4] Liu Yan. Brief Discussion on the Architectural Design for Middle and Small Housing, Scientific and Technological Information, 2013,(05) : 427. 